\title{
A New Perspective on "Community" and its Implications for Computer- Mediated Communication Systems
}

\section{Amy Bruckman}

College of Computing

$855^{\text {th }}$ St., TSRB 338

Georgia Institute of Technology

Atlanta, GA 30332-0760

asb@cc.gatech.edu
Copyright is held by the author/owner(s).

CHI 2006, April 22-27, 2006, Montréal, Québec, Canada. ACM 1-1-59593-298-4/06/0004

\begin{abstract}
Scholars have long argued about the nature of "community," and the growth of Internet-based communication and "online communities" has intensified this debate. This paper argues that a new perspective on the concept "community" can shed light on the subject. I deas from cognitive science, particularly category theory, can help. I suggest that community can be viewed as a prototype-based

category. Prototype-based categories are defined not by simple rules of inclusion and exclusion, but instead by their prototypical members-a robin is a better example of a bird than an emu or a penguin. Items in a category are better or worse examples of the category depending on their degree of similarity to the prototypical members. I will argue that these theoretical insights can help resolve debates about the nature of community, and also can help guide designers of computer-mediated communication (CMC) systems.
\end{abstract}

\section{Keywords}

Community, online communities, virtual communities, cognitive science, category theory. 


\section{ACM Classification Keywords}

H.5.3 Group and Organizational Interfaces;

Collaborative computing

\section{Angsting About Community}

Much ink has been spilled on the topic of whether online communities are "really communities." On the positive side, writers like Howard Rheingold make a compelling case for the value "online communities" can provide for members. Rheingold tells powerful stories of people on the bulletin board system The WELL providing one another with real aid in times of crisisfor example, helping to arrange medical evacuation for a member who became ill in rural India, or mailing books to a book-loving member who lost his library in a fire $[8, p .3]$

On the other hand, the word "community" is often used so casually that it becomes a synonym for "group." Kling and Courtright write that "casual use of the term community to characterize groups that are engaged in learning, or groups that participate in e-forums, is seriously misguided" [3, p. 91]. From this perspective, creating a successful online community is a substantial accomplishment, and using the word indiscriminately trivializes that achievement. Jenny Preece notes that "emotions can run high over this issue. Conference and journal reviewers have been known to reject papers because they felt use of the term community had been trivialized" [7, pp. 13-14]. Discussing whether groups on USENET are communities, Teresa Roberts argues that 'the word 'community' has been used in a metaphorical sense for long enough that it doesn't have a precise meaning" [9].
In this paper, I begin by reviewing the recent history of controversies about the nature of "community." Next, I propose a new perspective on the concept "community" based in cognitive science research about categories and how they operate in the mind and in language. Finally, possible practical implications for the analysis and design of CMC systems are suggested.

\section{Question that Predates Computer Networks}

It's not surprising that what constitutes an "online community" is contested, given that the underlying notion of "community" has always been hotly debated by sociologists $[2,12,14]$. Particularly since the invention of the automobile, sociologists have debated whether communities need to be geographically collocated. George Hillery analyzed 94 different definitions of community from the literature at the time of his writing in 1955, and concludes that most "are in basic agreement that community consists of persons in social interaction within a geographic area and having one or more additional common ties" [2]. In contrast, by 1979 the field of social network analysis was becoming prominent, and lending increasing evidence to support the idea that both weak and strong interpersonal ties can exist over distance-communities don't need to be geographically collocated. Barry Wellman and Barry Leighton make a compelling case that "neighborhood" and "community" are separate concepts, both important [14].

The rise of additional means of transportation and communication in latter parts of the $20^{\text {th }}$ century brought into closer focus a debate about the nature of "community" which was already longstanding. Wellman and Milena Gulia caution us that our more idealistic notions of community may be overblown. They write 
that, "pundits worry that virtual community may not truly be community. These worriers are confusing the pastoralist myth of community for reality. Community ties are already geographically dispersed, sparsely knit, connected heavily by telecommunications (phone and fax), and specialized in content" [13, p. 187].

It's worth noting that the word "community" is often used in a value-laden way. When researchers declare that a particular group is not a community, they typically are not just debating semantics, but passing a value judgment. However, it's unclear what these debates contribute to our understanding of the evolving genre of computer-mediated communication. I will argue that a new perspective on the concept "community" can help us focus our attention on more salient questions.

There are many possible ways to understand the concept "community," and these are by no means mutually exclusive. For example, John Carroll and colleagues make the intriguing proposal that the idea of "collective efficacy," is a useful measure of community [1]. No metric or perspective on the concept is the "correct" one. Rather, a variety of conceptual frameworks can be used in complimentary fashion to highlight different aspects of a complex phenomenon. This theoretical analysis is ultimately in the service of design-different definitions lead us to ask different questions, which ultimately can help guide designers.

\section{Community as a Category}

What really is a "community"? I suggest that cognitive science can help answer the question more clearly. I will argue that the word "community" refers to a category of associations of groups of people.
To understand "community," it helps to have a more nuanced view of a "category." Eleanor Rosch found that categories are not organized by simple rules of inclusion and exclusion, but by prototypes. Each category has one or more best or "focal" members. These are the prototypes for the category. For example, a robin or sparrow is a better example of a bird than an ostrich or penguin [10]. Thus, when Wellman and Gulia argue that the worriers are confusing a "pastoralist myth" for the reality of community, they are saying in effect that our focal members for the category community are ahistorical and idealized.

Within a category each item has a degree of membership. The degree of membership of an item in a category depends on its similarities and differences from the focal members [5]. Rosch notes, however, that talking about "the focal members" of a category is a linguistic convenience. It would be better instead to refer to the "degree of prototypicality" of each member of the category [10]. In experimental research in cognitive science, degree of prototypicality can be measured with reaction-time studies. A subject asked if a robin is a bird will respond much more quickly than when asked if a penguin is a bird [10, p. 198]. These results are generally consistent across individuals from a particular cultural background.

\section{Fuzzy Boundaries}

Categories can have either clear or fuzzy boundaries. For example, the categories "car" and "truck" have fuzzy boundaries, and sport-utility vehicles (SUVS) are members of both groups. However, SUVs are somewhat remote members of both the "car" and 
"truck" categories-a Ford Explorer is not an ideal example of either a car or a truck.

In this light, asking whether something "is a community" is a poorly formed question unlikely to yield deep insights. The category "community" has fuzzy boundaries. Instead, we can ask how similar a particular group is to our ideal models of community. This is a more productive line of inquiry, because it challenges us to reflect on the nature of our prototypical models of community, and explore in detail their specific features and why each feature might or might not matter.

Prototypes as Cultural Constructs

Prototypes (or degrees of prototypicality) for categories are in fact cultural constructs. This is true even for something as seemingly concrete and objective as the color of objects. Color perception is based on a set of focal colors, which vary by culture. English speakers recognize eleven focal colors, while some cultural/linguistic groups recognize as few as two.

What people from one culture will call a shade of blue, those from another culture may consistently call a shade of green [5, pp. 24-31]. If something as seemingly basic as color perception varies by culture, a phenomenon as slippery as the ideal of "community" varies much more dramatically.

The concept of community clearly varies strongly between cultures, but it remains an open question how much it varies within each culture. Prototypes tend to have regularity across individuals within a group, as reaction time studies show. In other words, English speakers tend to agree that a desk chair is a pretty typical chair, and a rocking chair less so. This opens the intriguing question: is there significant regularity in the concept of community across individuals, or is this idea more idiosyncratic? Empirical investigation of this question has not yet been undertaken.

\section{Radial Categories and Genre}

Many concepts resist definition with one set of prototypes. For example, Lakoff details how complicated it is to define the category "mother." Beyond the central case of "a mother who has always been female, and who gave birth to the child, supplied her half of the child's genes, nurtured the child, is married to the father, is one generation older than the child, and is the child's legal guardian" [5, p. 83], we must also account for the idea of a stepmother, adoptive mother, birth mother, etc. He notes that "the point is that the central case does not productively generate all these subcategories. Instead, the subcategories are defined by convention as variations on the central case." The category "mother" is, in Lakoff's terminology, a "radial category" [5, p. 83-84].

Similarly, community is also a radial category. Our notion of the basic concept "community" for many conjures up idealized images of small-town life. However, the base-level concept does not explain subcategories like corporations, work groups, army platoons, and daycare centers. A corporation has many aspects that can be understood through the lens of the idea of community. How do employees support one another? What are the patterns of social relation that emerge among them? Can employees successfully leverage both strong and weak ties to others in the organization to help them accomplish goals? These are excellent questions to ask-the idea of community can be a powerful one for understanding organizations. 
However, it's more productive to ask "How is this corporate community like and unlike IBM?" than "How is this like and unlike the town square?" We can better understand this example within the subcategory. We can think of the subcategories as genres of community. In $\mathrm{HCl}$ design work, we need to understand our deliberately engineered social groups each within their intended genre(s).

\section{New Salient Questions}

Our task here is to apply these ideas from sociology and cognitive science in the service of design. To design a computer-mediated communication system with community-like aspects, I propose that we begin by asking a series of questions:

1. What kind(s) of community are we concerned with?

2. What are our implicit prototypes for each relevant subcategory of community?

3. What are the most salient characteristics of those prototypes?

4. Are there alternate prototypes we should consider?

5. How can we learn from our prototypes and alternate prototypes to guide our design process?

This is an iterative process-one may need to return to previous steps often. In most cases, one will need to analyze a design from multiple points of view. For example, those seeking to create an online, free-time educational site for kids may examine it both as a learning community, and a third place (a social environment that is neither work nor home [6]). Design work can then draw on features of both of these models.
The simple act of trying to name our implicit prototypes and reflect on them can often reveal hidden assumptions to challenge. For example, when asked to think about a learning community, many people think of the traditional classroom. However, much of the field of education is devoted to questioning our assumptions about that learning environment.

Recognizing this fact, before we model a new distance learning environment on the idea of a teacher lecturing and students taking notes, we might ask basic questions about whether that is the most effective method of teaching and learning.

Some of the most innovative design work is grounded in the creative selection of alternative prototypes. For example, Marlene Scardamalia and Carl Bereiter

suggest that perhaps an alternate model for a learning community is a community of scientists. Through peer review and publication, academic scholars collaboratively build knowledge, forming a "knowledgebuilding community." Scardamalia and Bereiter suggest that a knowledge-building community might be a novel context to help children learn [11]. Their successful CSILE software was designed with this alternative prototype as the guide.

\section{Conclusion}

Viewing "community" as a prototype-based category helps resolve some of the emotionally charged debates about the nature of community that have persisted in the literature about the design of CMC systems. Additionally, this perspective on community leads us to ask different questions. In doing empirical fieldwork, understanding prototypical models can help us to analyze existing systems more insightfully. In the design process, understanding existing models can help 
us to benefit from past experiences of others and better leverage existing design patterns and heuristics. By identifying our implicit mental models for group interaction, we can uncover hidden assumptions that may be challenged. By identifying alternate models, we may support creative leaps in design.

This theoretical analysis suggests a number of directions for future work. It would be productive to empirically study existing genres of community in particular cultures, and better understand their unique qualities. Additionally, research on design method could productively explore how understanding different genres of community can aid the design process for CMC systems.

\section{Acknowledgments}

Barry Wellman and Nancy Nersessian provided invaluable help. Special thanks to Jason Elliott, Andrea Forte, Jim Hudson, and J osé Zagal.

\section{References}

1. Carroll, J.M., M.B. Rosson, and J. Zhou. Collective Efficacy as a Measure of Community. In CHI 2005. Portland, OR. ACM Press, pp. 1-10.

2. Hillery, G.A., Definitions of community: areas of agreement. Rural Sociology, 1955. 20 (J une): pp. 111-123.

3. Kling, R. and C. Courtwright, Group Behavior and Learning in Electronic Forums. In Designing for Virtual Communities in the Service of Learning, S. Barab, R. Kling, and J. Gray, Eds. Cambridge University Press, New York, 2004, pp. 91-119.
4. Lakoff, G., Cognitive Models and Prototype Theory. In Concepts, E. Margolis and S. Laurence, Eds. MIT Press, Cambridge, MA, 1999, pp. 391-421.

5. Lakoff, G., Women, Fire, and Dangerous Things; What Categories Reveal about the Mind. University of Chicago Press, Chicago, 1987.

6. Oldenburg, R., The Great Good Place. Paragon House, New York, 1989.

7. Preece, J., Online Communities: Designing Usability, Supporting Sociability. John Wiley \& Sons, New York, 2000.

8. Rheingold, H., The Virtual Community: Homesteading on the Electronic Frontier. AddisonWesley, Reading, MA, 1993.

9. Roberts, T. Are Newsgroups Virtual Communities? In CHI 1998. LA, CA. ACM Press, pp. 360-367.

10. Rosch, E., Principles of Categorization. In Concepts, E. Margolis and S. Laurence, Editors. MIT Press, Cambridge, MA, 1999, p. 189-206.

11. Scardamalia, M. and C. Bereiter, Computer Support for Knowledge-Building Communities. Journal of the Learning Sciences, 1994. 3(3): pp. 265-283.

12. Schnore, L.F., Community. In Sociology: An Introduction, N.J. Smesler, Editor. John Wiley \& Sons, New York, 1967, pp. 79-150.

13. Wellman, B. and M. Gulia, Virtual Communities are Communities: Net Surfers Don't Ride Alone. In Communities in Cyberspace, M.A. Smith and P. Kollock, Editors. Routledge, New York, 1999.

14. Wellman, B. and B. Leighton, Networks, Neighborhoods, and Communities: Approaches to the Study of the Community Question. Urban Affairs Quarterly, 1979. 14(3): pp. 363-390. 